\title{
RESEARCH
}

Open Access

\section{Biological and clinical impact of central nervous system hemangioblastomas in Chinese patients with von Hippel-Lindau disease: implications for treatment}

Zhen Liu', Liang $\mathrm{Li}^{1 *+} \mathbb{D}$, Zhiqiang $\mathrm{Yi}^{1+}$, Hongzhou Duan ${ }^{1}$, Runchun Lu${ }^{1}$, Chunwei $\mathrm{Li}^{1}$, Lei Li ${ }^{2}$ and Kan Gong ${ }^{2}$

\begin{abstract}
Objective: Central nervous system (CNS) hemangioblastomas (HGBs) are the most frequent cause of mortality in patients with von Hippel-Lindau (VHL) genetic syndrome. However, there is a lack of large studies on the clinical features and optimal management of HGBs in Chinese patients.

Methods: VHL-related HGB cases treated surgically at our hospital from 2012 to 2019 were evaluated. Patients and family members meeting the clinical diagnostic criteria underwent genetic testing. Clinical, genetic and relevant imaging data were analyzed.

Results: Eighty-five VHL patients from 34 pedigrees in 16 Chinese provinces who underwent 121 operations for CNS HGBs were enrolled. Multiple operations were associated with a younger age at first operation $(\mathrm{OR}=0.926$, 95\% Cl=0.871-0.985, $P=0.014$, threshold: 27.5 , sensitivity: $72.2 \%$, specificity: $71.2 \%$ ) and a longer postoperative period ( $\mathrm{OR}=1.096,95 \% \mathrm{Cl}=1.015-1.184, P=0.019$, threshold: 10.5 , sensitivity: $66.7 \%$, specificity: $76.3 \%)$. The age at first operation was younger in children than in their parents (23 pairs, $P<0.001$ ). The age at first operation was younger in siblings born later than in those born earlier (10 pairs, $P=0.01$ ). Most untreated tumors (98.2\%) remained relatively stable during follow-up (range, $0.5-7$; median, 2). However, new tumors continued to emerge (0.14 tumor/year).

Conclusion: VHL-associated CNS HGB is a long-term chronic disease with repeated attacks, likely with genetic anticipation in Chinese pedigrees. When the age at first operation is under 27.5 years, or the postoperative period is longer than 10.5 years, the risk of multiple operations is increased. While most unresected HGBs remain stable after surgery, new tumors may still slowly emerge; hence, scheduled follow-ups are necessary.
\end{abstract}

Keywords: VHL genetic syndrome, Genetic mutation, Hemangioblastoma surgical treatment, Genetic anticipation

\footnotetext{
* Correspondence: doctzyf@126.com

${ }^{+}$Liang Li and Zhiqiang Yi contributed equally to this work.

'Department of Neurosurgery, Peking University First Hospital, No. 8 Xishiku Street, Xicheng District, Beijing 100034, China

Full list of author information is available at the end of the article
}

C C The Author(s). 2020 Open Access This article is licensed under a Creative Commons Attribution 4.0 International License, which permits use, sharing, adaptation, distribution and reproduction in any medium or format, as long as you give appropriate credit to the original author(s) and the source, provide a link to the Creative Commons licence, and indicate if changes were made. The images or other third party material in this article are included in the article's Creative Commons licence, unless indicated otherwise in a credit line to the material. If material is not included in the article's Creative Commons licence and your intended use is not permitted by statutory regulation or exceeds the permitted use, you will need to obtain permission directly from the copyright holder. To view a copy of this licence, visit http://creativecommons.org/licenses/by/4.0/ The Creative Commons Public Domain Dedication waiver (http://creativecommons.org/publicdomain/zero/1.0/) applies to the data made available in this article, unless otherwise stated in a credit line to the data. 
Von Hippel-Lindau (VHL) genetic syndrome is an autosomal dominant genetic disease caused by pathogenic variants in the VHL tumor suppressor gene on chromosome 3 (3p25-26). It consists of three exons and two introns within a $10 \mathrm{~kb}$ region [1,2]. This disease carries a prevalence of 1 in 36,000 births and is highly penetrant (>90\% penetrance by 65 years of age) $[3,4]$. The $V H L$ gene encodes a tumor suppressor protein (pVHL) that regulates hypoxia-inducible factor (HIF) protein. The main reason for VHL genetic syndrome is $\mathrm{PVHL}$ inactivation caused by genetic mutations, resulting in dysfunction in regulating the proteolytic degradation of HIF [5]. Uncontrolled HIF expression increases the expression of a wide range of target genes involved in angiogenesis, proliferation and metabolism, including vascular endothelial growth factor (VEGF) and C-X-C motif chemokine receptor 4 (CXCR4). VEGF overexpression is a leading factor in tumor angiogenesis, especially for hemangioblastomas (HGBs) [6, 7]. The main manifestation of VHL syndrome in the central nervous system (CNS) is HGB. Although HGB is a benign tumor, its cystic characteristics and associated peritumoral edema often lead to neurological morbidity and mortality [8]. Additionally, its natural history and management have not been defined. In recent studies, researchers have considered the tumor burden of VHL-associated HGBs to be associated with partial germline mutations, male sex and a younger age [9-11]. Most of these studies were performed in European and North American countries. There is a lack of large studies on the clinical features, pathogenic variants and optimal management of HGBs in Chinese patients. Therefore, we retrospectively analyzed patients with VHL-associated CNS HGBs admitted between 2012 and 2019. The patients and family members who met the clinical diagnostic criteria underwent genetic testing. Clinical, genetic and relevant imaging data were analyzed.

\section{Methods}

\section{Patients}

This study was approved by the ethics committee of our hospital. All patients involved in this study provided informed consent. From June 2012 to May 2019, 37 patients with VHL genetic syndrome confirmed by genetic diagnosis underwent surgery for CNS HGBs at our hospital, and all cases were verified by postoperative pathology. These patients who met the clinical diagnostic criteria and their family members underwent genetic testing. The diagnosis was established when the patient was found to carry a germline $V H L$ mutation or fulfilled the previously described clinical criteria. Because of the late onset in some patients, their biological parents were deceased and not available for genetic testing. At least one patient was diagnosed via $V H L$ mutation testing in each family; Clinical data including sex, family history, mutation type, onset age of each organ, the cause of death and relevant imaging data were obtained by reviewing medical records or interviewing family members. Fiften patients were excluded from the study due to unclear data. A total of $85 \mathrm{VHL}$ patients from 34 pedigrees in 16 Chinese provinces who underwent 121 operations for CNS HGBs were enrolled.

\section{Germline genotype analysis}

Genomic DNA was extracted from peripheral blood using the QIAamp DNA Blood Mini Kit (QIAGEN, Germany). Three coding exons and their flanking intronic regions were amplified by polymerase chain reaction (PCR) using primers as described previously [12]. Direct sequencing was used to detect missense and splicing mutations. Large exon deletions were detected using a multiplex ligation-dependent probe amplification kit (MLPA, P016-C2, MRC-Holland, Amsterdam) and confirmed by real-time quantitative PCR using the primers described by Ebenazer et al. [13]. All patients were divided into the following five groups: missense group, partial deletion group, large deletion group, frameshift group and splicing site mutation group.

\section{Clinical assessment and imaging analysis}

All surgical patients underwent contrast-enhanced MRI (T1-weighted imaging (T1WI), T2-weighted imaging, diffusion-weighted imaging and contrast-enhanced T1WI; slice thickness, $1 \mathrm{~mm}$ ) examinations to determine the lesion location. The volume of the tumor and associated cysts was calculated using a modified ellipsoid formula (volume $=$ largest anteroposterior diameter (centimeter)*largest mediolateral diameter (centimeter)*largest craniocaudal diameter (centimeter)* 0.5$)$ [14]. Abdominal computed tomography and fundoscopy were performed in all patients to identify related lesions in other target organs. The Karnofsky Performance Scale (KPS) score was used to evaluate neurological function, and postoperative follow-up examinations were performed every 6 months. Patients underwent follow-up contrast-enhanced MRI examinations to detect the presence of unresected tumor or recurrence.

\section{Surgical technique}

Surgery was performed on patients who had tumors with obvious mass effects on MRI. Because HGBs are usually located in the posterior and medial portions of the cerebellum, brainstem and spinal cord, we often used the posterior median approach for surgical resection. For tumors located anteriorly, laterally or at other sites, we selected the appropriate surgical approach based on optimum visualization and direct access to the tumor. The process of tumor resection was typically performed 
as follows: First, we separated the tumor from the surrounding tissue. Then, we gradually severed the feeding arteries and drainage veins. Finally, we completely removed the tumor. For tumors with high-pressure peritumoral cysts, we released the cystic fluid to relieve the pressure and then removed the tumor. After complete tumor removal, the cyst was reexamined for residual tumor. For large cysts, we used endoscopy to explore the cysts to avoid missing tumors. Preoperative embolization was not performed in any patient.

\section{Statistical analysis}

Patients and tumor characteristics were analyzed using descriptive statistics. The difference in the number of operations in each variant group was compared using the Mann-Whitney $U$ test. The chi-squared test was used to compare the sex distribution between two groups. The significance of associations with the outcome of multiple operations was evaluated using univariate logistic analysis. Then, multivariate analysis was used to analyze those significant variables as independent predictors for multiple operations. Receiver operating characteristic (ROC) curve analysis was used to evaluate the predictive value. The optimal cutoff value was calculated by Youden's index. Differences between generations and peers were analyzed using the Wilcoxon test.

\section{Results}

\section{Patient characteristics}

A total of $85 \mathrm{VHL}$ patients from 34 pedigrees in 16 Chinese provinces who underwent 121 operations for CNS HGBs were enrolled. The mean age at first operation in these patients was $33.9 \pm 13.6$ years (range, 1374; median, 30). The male-to-female ratio was 1.02 (males, 43; females, 42). Genetic analysis showed that there were 13 families in the partial deletion group, 3 families in the large deletion group, one family in the frameshift group and 15 families in the missense group (Table 1). Variants within exons appeared in 32 pedigrees. Two types of splicing stie variants appeared in 2 families. Because of the late onset in some patients, their biological parents were deceased and not available for genetic testing. However, 8 (8/33, 24\%) of the remaining patients exhibited de novo mutations confirmed by paternity testing. According to the American College of Medical Genetics and Genomics standards and guidelines, we found that there were 68 pathogenic variants and 17 likely pathogenic variants among these patients.

Eight patients died from aspiration and infection after the first operation. The remaining 77 patients (males, 38; females, 39 ; age, $43 \pm 14.7$; range, $18-76$; median, 43 ) recovered well after the first operation and survived until December 2019. Forty-five of the 77 patients in the deletion group (including partial deletions, large deletions and a frameshift group) underwent 66 operations for CNS HGB, and 33 patients in the missense group underwent 48 operations. Our statistical results showed no significant difference in sex, age at first operation or number of operations among five kinds of variants (Table 1). The chi-square test results showed no significant difference in the number of operations between the deletion group and missense group $(P=0.824)$. In the multivariate analysis, only two covariates remained in the final model: the age at first operation $(\mathrm{OR}=0.926$, 95\% $\mathrm{CI}=0.871-0.985, P=0.014<0.05)$ and the period after the first operation $(\mathrm{OR}=1.096,95 \% \mathrm{CI}=1.015-$ $1.184, P=0.019<0.05)$. The probability of reoperation was calculated using the following formula: $\log (\mathrm{p} / 1-\mathrm{p})=$ $0.092 \times 1+0.077 \times 2\left(\chi_{2}=15.462 \quad P<0.001\right) \quad($ Table 2$)$. Model performance was used to select thresholds to discriminate patients by age at first operation and period after the first operation. As shown in Fig. 1, the area under the ROC curve (AUC) of the selected model for age at first operation was $0.737(95 \% \mathrm{CI}=0.624-0.831$, standard error: 0.069 ). The optimal threshold was 27.5 (sensitivity: $72.2 \%$, specificity: $71.2 \%$, Youden's index: 0.434). The AUC of the selected model for the period after the first operation was $0.718(95 \% \mathrm{CI}=0.575$ 0.860, standard error: 0.073), and the optimal threshold was 10.5 (sensitivity: 66.7\%, specificity: 76.3\%, Youden's index: 0.43). The difference between the two AUCs was not significant $(P=0.84)$. In addition, genealogical analysis showed that the age at first operation was younger

Table 1 Patient characteristics in different variants type

\begin{tabular}{lllllll}
\hline & Families & HGB patients(total) & HGB patients (alive) & Male & First operation age in HGB & $\begin{array}{l}\text { The average times of } \\
\text { operation in HGB }\end{array}$ \\
\hline Partial deletion & 13 & 35 & 33 & 23 & $32.8 \pm 14.3$ & $1.3 \pm 0.8$ \\
Large deletion & 3 & 12 & 10 & 5 & $34.8 \pm 12.8$ & $1.8 \pm 1.4$ \\
Frameshift & 1 & 1 & 1 & 1 & 39 & 1 \\
Missense & 15 & 31 & 27 & 20 & $33 \pm 12.3$ & $1.3 \pm 0.8$ \\
Splicing site mutation & 2 & 6 & 6 & 2 & $40.2 \pm 19.1$ & $1.7 \pm 1.2$ \\
total & 34 & 85 & 77 & -43 & $33.7 \pm 13.6$ & $1.4 \pm 0.93$ \\
$P$ & - & - & - & 0.593 & 0.716 & 0.628 \\
\hline
\end{tabular}


Table 2 Analysis of factors associated with number of operations

\begin{tabular}{lll}
\hline Variables & $\boldsymbol{P}$ value & OR,95\% Cl \\
\hline Sex & 0.254 & - \\
Mutation & 0.261 & - \\
Age at first operation & 0.14 & $0.926,0.871-0.985$ \\
Period after the first operation & 0.19 & $1.096,1.015-1.184$ \\
\hline
\end{tabular}

in children than in their parents (23 pairs, $P<0.001$ ). This phenomenon might be related to genetic anticipation. The age at first operation was younger in siblings born later than those born earlier (10 pairs, $P=0.01<$ 0.05).

\section{Tumor characteristics}

Because some of the patients did not undergo surgery at our hospital, to accurately assess the tumor burden, distribution and development, we analyzed the clinical and relevant imaging data from only patients who underwent surgery at our hospital. The mean operative age of the 37 patients treated at our hospital was $36.3 \pm 13.6$ years (range, 20-67 years; median, 34 years), the male-tofemale ratio was 0.95 (males, 18; females, 19), and the mean follow-up duration was $2.24 \pm 1.71$ years (range, 0.5-7 years; median, 2 years). Preoperative MRI showed that 37 patients harbored 182 CNS HGBs, with an average of $4.92 \pm 2.92$ tumors/patient (range, 1-13; median, 4). The tumor sites were as follows: 66 tumors (36\%), right cerebellum; 52 tumors (29\%), left cerebellum; 8 tumors (4\%), cerebellar vermis; 19 tumors (10\%), medulla oblongata; 23 tumors (13\%), cervical spinal cord; and 14 tumors, other sites (Fig. 2). The major symptoms of HGBs in the cerebellum are headache (40.5\%) and gait ataxia (45.9\%). Symptomatic HGBs in the brainstem could lead to hydrocephalus (45.9\%) causing intracranial hypertension. Symptomatic tumors in the spine and cauda equina could lead to pain or urinary/bowel abnormalities. Out of the enrolled patients, only one patient with a symptomatic tumor of the thoracic spine underwent surgical intervention.

Thirty-seven patients underwent 40 operations at our hospital; 71 CNS HGBs were removed, with an average of $1.78 \pm 1.02$ tumors/patient, all of which were confirmed by postoperative pathology (range, 1-6; median, 2 ). Of these tumors, 51 were located in the cerebellum, 6 were located in the cervical spinal cord, 1 was located in the thoracic cord, and 13 were located in the medulla oblongata. Thirty-one cystic tumors were resected, including tumors with peritumoral and intratumoral cysts. Most of the cysts were located in the dorsal part near the midline. Thirty cystic lesions were located in the cerebellum, and 1 was located in the cervical spinal cord. The mean volume of cystic lesions resected from the cerebellum was $12.6 \pm 10.1 \mathrm{~cm}^{3}$. All resected tumors in the medulla oblongata were solid. Due to the obvious mass effects in the medulla oblongata, these $7 \mathrm{HGBs}$ were resected to avoid aggravating the hydrocephalus. The mean volume of tumors in the medulla oblongata was $9.2 \pm 7.3 \mathrm{~cm}^{3}$. Most of the other tumors were removed in the same operative field. Although the space occupying effects were not obvious, they were near those of the larger tumors. To prevent the postoperative volume from increasing, the other tumors were concurrently removed. Tumors not in the same operative area and without obvious occupying effects were not treated at the same time.

Thirty-six patients recovered well postoperatively and were discharged approximately 1 week later. One patient died from aspiration after surgery. Because of the high recurrence and low growth rate, our follow-up interval was 6 months. The mean follow-up duration in these 36

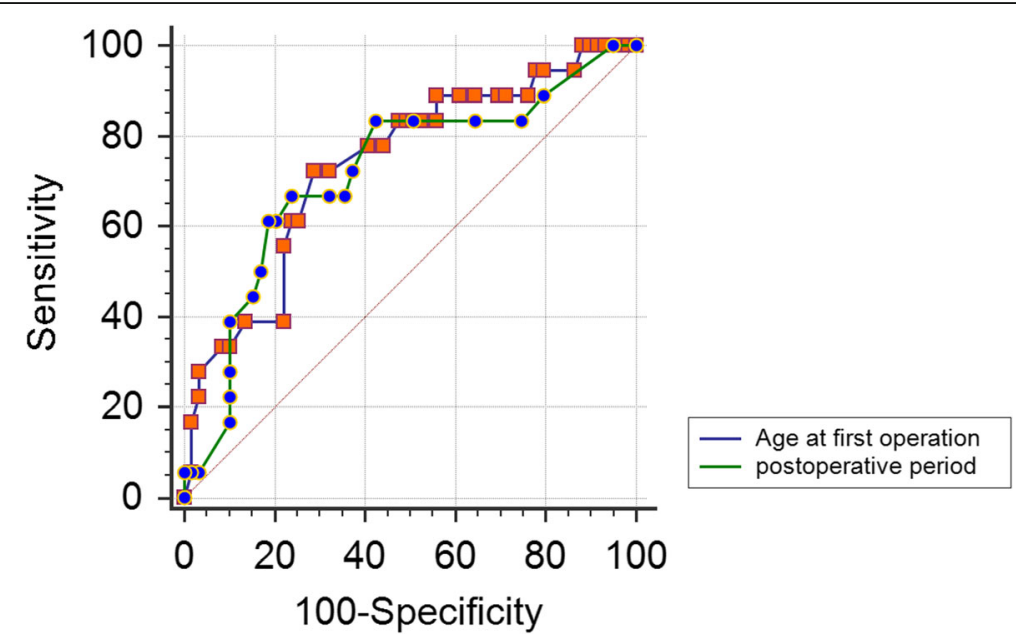

Fig. 1 ROC curve for multiple operations according to the age at first operation and the period after the first operation 


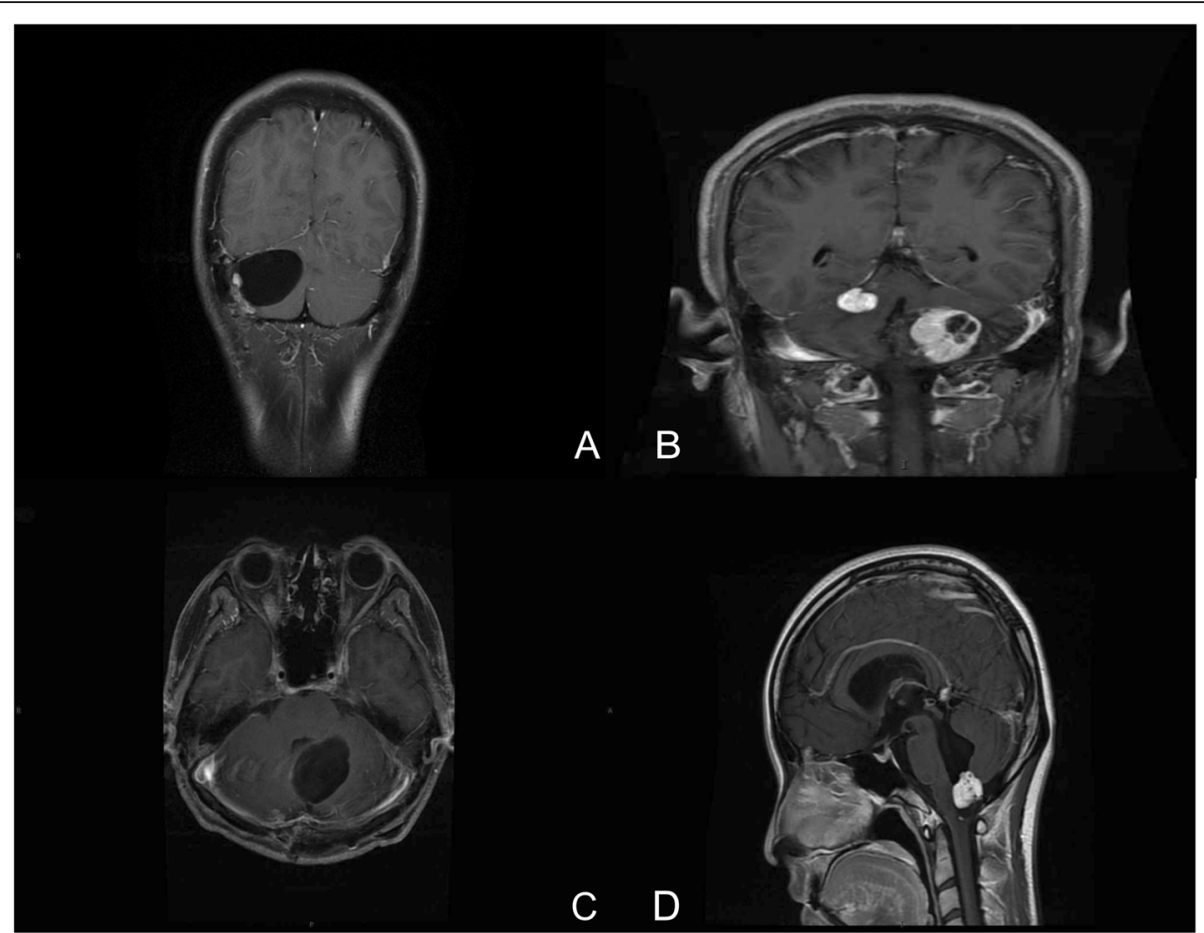

Fig. 2 Postcontrast MRI of cerebellar HGB and peritumoral cyst (a and $\mathbf{c}$ ), solid cerebellar tumor and intratumoral cyst (b), and HGB in the medulla oblongata (d)

patients was $2.24 \pm 1.71$ years (range, 0.5-7; median, 2). The KPS score at 6 months postoperatively was $94.7 \pm$ 7.7 (range, 80-100; median, 100). Postoperative reexamination showed that 109 (98.2\%) of the remaining $111 \mathrm{tu}-$ mors were in a stable state. Tumor enlargement was found in only 2 cases (1.8\%): one was located in the thoracic spinal cord, with obvious spinal cord compression, and reoperation was performed; the other was in the cerebellum without obvious symptoms, and this patient continued to be followed by observation. In addition, 10 new cerebellar HGBs appeared in 5 patients, for a recurrence rate of 0.14 tumors/year. Among the 37 patients, $81.1 \%$ had pancreatic cysts, $48.6 \%$ had renal cysts, and $48.6 \%$ had renal cancer. Pheochromocytomas were found in $13.5 \%$ of patients. Retinal HGBs occurred in $27 \%$ of patients. Thirty-seven patients underwent a total of 115 operations for renal cancer, pheochromocytomas, retinal HGBs and CNS HGBs, with an average of $3.1 \pm 1.59$ operations/patient (range, $1-7$; median, 3 ).

\section{Discussion}

\section{Biological and clinical implications}

Due to the pathogenic variants in $V H L$, CNS HGBs might manifest as a long-term chronic disease with repeated attacks occurring in VHL patients. In our study, 85 of the 99 VHL patients $(85.8 \%)$ from 34 pedigrees in 16 Chinese provinces underwent surgery for CNS HGBs. These 85 patients underwent a total of 121 operations for CNS HGBs (with a mean (SD) number of operations per patient of $1.47(0.97))$. These findings are consistent with the results of other research. In reports from the United States and Italy, over $80 \%$ of VHL patients develop CNS HGBs during their lifetime, and more than 90\% of patients with VHL-related HGBs develop multiple CNS HGBs $[15,16]$.

According to the public Human Gene Mutation Database (HGMD: http://www.hgmd.org), 627 variants have been identified. Missenses and partial deletions are the most common pathogenic variants in VHL. In our investigation, of 99 patients from 34 different families, 56\% showed deletions, and $44 \%$ showed missenses; 24\% (8/ 33) of these mutations were identified as de novo mutations, similar to the results reported by the NIH. We found no significant difference between the deletion groups (17 families, 44 HGB patients) and the missense groups (15 families, $33 \mathrm{HGB}$ patients) in the number of operations $(P=0.876)$, the number of CNS HGBs $(P=$ 0.208 ) or tumor size (cerebellum, $P=0.643$; medulla oblongata, $P=0.380$ ). The number of operations for renal cell carcinoma $(\mathrm{RCC})(P=0.827)$ or retinal angioma (RA) $(P=0.285)$ were also not significantly different between the deletion and missense groups. However, some studies have considered that partial deletions are associated with a higher risk of developing a large number of CNS HGBs [9, 17]. Perhaps this is because the protein produced by missense germline alterations can 
maintain intrinsic function in the context of neoplasias and metabolic syndromes, including $V H L[18,19]$. However, other studies have shown that deletions might be related to a lower risk of RCC and RA development in VHL patients [20]. Furthermore, Franke et al. observed that germline deletions were associated with a higher risk of RA development [17]. However, according to the present findings, there can be no unified conclusions regarding the exact mechanisms underlying some VHL manifestations. For this reason, it is difficult to determine the relationship between mutations and tumorigenesis. Further research is required to determine the mechanisms by which $V H L$ pathogenic variants influence phenotypes.

In addition, the progression of CNS HGBs was related to a younger age; our results reveal that age is a risk factor for tumor progression. A younger age at first operation for CNS HGBs results in a higher risk of reoperation. According to our statistical analysis, an age at first operation younger than 27.5 years predicted reoperation with a sensitivity and specificity of 72.2 and $71.2 \%$, respectively. Additionally, a longer period after surgery was associated with a higher chance of reoperation; a period longer than 10.5 years predicted reoperation for CNS HGBs, with a sensitivity and specificity of 66.7 and $76.3 \%$, respectively. Some studies have found that younger patients are much more likely to develop more tumors per year than older patients [9]. Hence, the early resection and close follow-up of symptomatic CNS HGBs are very important for patients with a younger age of onset. Simultaneously, the results from the family study show that the age at first operation for CNS HGBs was younger in children than in their parents (23 pairs, $P=0.0<0.001)$. The regularity with which this occurs might be associated with genetic anticipation. The reasons for this phenomenon are considered to be related to telomere shortening in the offspring [21]. Our results also show that the age at first operation was younger in siblings born later than in those born earlier. The cause of this phenomenon remains unclear. Some scholars have considered the cause to be related to hormone exposure during pregnancy [22].

\section{Tumor burden and treatment}

Most VHL patients (92\%) often have multiple CNS HGBs resected when undergoing surgery on the nervous system, with an average of $4.92 \pm 2.92$ CNS HGBs/patient. More than half (67\%) of the tumors were located in the cerebellum. The chief mechanism responsible for the symptomatology is the cystic changes in solid tumors. Nearly $84 \%$ of patients underwent surgery due to the mass effects caused by cystic tumor changes. Some studies have considered that cystic tumors grow faster than the mean associated tumor growth rate and that symptoms caused by cysts in highly eloquent areas may be related to early cyst progression [23]. The mechanism of peritumoral cyst formation mainly involves high intratumoral pressure and increased vascular permeability [24]. Currently, the treatment of cystic tumors is primarily surgical resection. Recent studies showed that radiotherapy for peritumoral cysts achieves limited effects and could result in peritumoral edema or even cyst progression [25]. Therefore, early surgical resection seems to be an effective therapy for symptomatic CNS HGB cysts. Furthermore, our results show that solid tumors located in the medulla oblongata are more likely to lead to hydrocephalus in the early stage. However, the very complex areas and abundant vascular networks make tumor resection difficult. Approximately $10 \%$ of the tumors were located in the medulla. Additionally, $32 \%$ of medullary HGBs were resected due to hydrocephalus. The mean volume of tumors resected from the medulla oblongata was $9.2 \pm 7.3 \mathrm{~cm}^{3}$. Because of the abundant blood supply in the tumor, some surgeons apply preoperative embolization to reduce the risk of bleeding. However, none of the 37 patients treated at our hospital underwent preoperative embolization. Only 2 patients (5\%) received intraoperative blood transfusions, and postoperative hemorrhage was not observed in any patient. Except for one patient who died from aspiration, all 36 patients recovered well, with no complications. The 6-month postoperative follow-up yielded a mean KPS score of $94.7 \pm 7.7$ (range, 80-100; median, 100). Recent studies have also reported that preoperative embolization did not decrease the blood loss volume or incidence of postoperative complications; additionally, preoperative embolization fails to improve the gross total resection rate, and the embolization procedure carries a significant risk for complications [26-28]. Postoperative reexaminations revealed that most tumors $(98.2 \%)$ were relatively stable, with only 2 tumors increasing in volume. Ten new-onset cerebellar HGBs were found in 13.5\% of the patients, showing an average new tumor rate of 0.14 tumors/year. Considering the growth pattern of CNS HGBs, it is not necessary for patients to undergo frequent follow-up examinations; the followup interval for CNS HGBs should be 1 year or 6 months.

\section{Conclusion}

VHL-related CNS HGB is a long-term chronic disease with repeated attacks, likely with genetic anticipation in Chinese pedigrees with VHL-related HGBs. The age at first operation is younger in siblings born later than in those born earlier. Symptoms of HGBs are mainly 
related to the mass effects caused by cystic changes, and the most common site is the cerebellum. When the age at first operation is under 27.5 years or the operation interval is longer than 10.5 years, the risk of multiple operations is increased. Most unresected HGBs are stable after surgery; however, new tumors may continue to emerge. Hence, scheduled follow-up examinations are necessary.

\section{Financial disclosures}

Peking University First Hospital Cross Clinical Study Foundation; Youth Clinical Research Project of Peking University First Hospital (2019CR02).

\section{Authors' contributions}

ZL: Designed and conceptualized study, analyzed the data, and drafted the manuscript for intellectual content. LL: Data collection and analysis, drafting and revision of the manuscript, drafted the manuscript for intellectual content. ZY: Data collection and statistical analysis, drafting and revision of the manuscript. HD: Data collection and statistical analysis, drafting and revision of the manuscript. RL: Helped perform the analysis through constructive discussions. CL: Helped perform the analysis through constructive discussions. LL: Performed the experiments and conducted the genetic sequencing. KG: Drafted the manuscript for intellectual content. All authors read and approved the final manuscript.

\section{Funding}

Not applicable.

\section{Availability of data and materials \\ Not applicable.}

\section{Ethics approval and consent to participate}

All procedures performed in studies involving human participants were in accordance with the ethical standards of the institutional and/or national research committee and with the 1964 Declaration of Helsinki and its later amendments or comparable ethical standards. Informed consent was obtained from all individual participants included in the study.

\section{Consent for publication}

Not applicable.

\section{Competing interests}

The authors certify that there is no conflict of interest with any individual/ organization for the present work.

\section{Author details}

'Department of Neurosurgery, Peking University First Hospital, No. 8 Xishiku Street, Xicheng District, Beijing 100034, China. ${ }^{2}$ Department of Urology, Peking University First Hospital, Beijing 100034, China.

\section{Received: 8 January 2020 Accepted: 19 October 2020}

\section{Published online: 22 October 2020}

\section{References}

1. Richards FM, Maher ER, Latif F, Phipps ME, Tory K, Lush M, et al. Detailed genetic mapping of the von Hippel-Lindau disease tumour suppressor gene. J Med Genet. 1993;30(2):104-7. https://doi.org/10.1136/jmg.30.2.104.

2. Kuzmin I, Duh FM, Latif F, Geil L, Zbar B, Lerman MI. Identification of the promoter of the human von Hippel-Lindau disease tumor suppressor gene. Oncogene. 1995;10(11):2185-94.

3. Neumann $\mathrm{H}$, Wiestler O. Clustering of features of von Hippel-Lindau syndrome: evidence for a complex genetic locus. Lancet. 1991;337(8749): 1052-4. https://doi.org/10.1016/0140-6736(91)91705-Y.

4. Maher E, Iselius L, Yates J, Littler M, Benjamin C, Harris R, et al. Von HippelLindau disease: a genetic study. J Med Genet. 1991;28(7):443-7. https://doi org/10.1136/jmg.28.7.443.

5. Jaakkola P, Mole DR, Tian YM, Wilson MI, Gielbert J, Gaskell SJ, et al. Targeting of HIF-alpha to the von Hippel-Lindau ubiquitylation complex by O2-regulated prolyl hydroxylation. Science. 2001;292(5516):468-72.
6. Kruizinga RC, van Marion DM, den Dunnen WF, de Groot JC, Hoving EW, Oosting SF, et al. Difference in CXCR4 expression between sporadic and VHL-related hemangioblastoma. Familial Cancer. 2016;15(4):607-16. https:// doi.org/10.1007/s10689-016-9879-3.

7. Pierscianek D, Wolf S, Keyvani K, El Hindy N, Stein KP, Sandalcioglu IE, et al. Study of angiogenic signaling pathways in hemangioblastoma. Neuropathology. 2017;37(1):3-11. https://doi.org/10.1111/neup.12316.

8. Butman J, Linehan W, Lonser R. Neurologic manifestations of von HippelLindau disease. JAMA. 2008;300(11):1334-42. https://doi.org/10.1001/jama. 300.11.1334.

9. Lonser R, Butman J, Huntoon K, Asthagiri A, Wu T, Bakhtian K, et al. Prospective natural history study of central nervous system hemangioblastomas in von Hippel-Lindau disease. J Neurosurg. 2014;120(5): 1055-62. https://doi.org/10.3171/2014.1.JNS131431.

10. Ye DY, Bakhtian KD, Asthagiri AR, Lonser RR. Effect of pregnancy on hemangioblastoma development and progression in von Hippel-Lindau disease. J Neurosurg. 2012;117(5):818-24. https://doi.org/10.3171/2012.7. jns12367.

11. Vortmeyer AO, Gnarra JR, Emmert-Buck MR, Katz D, Linehan WM, Oldfield $\mathrm{EH}$, et al. von Hippel-Lindau gene deletion detected in the stromal cell component of a cerebellar hemangioblastoma associated with von HippelLindau disease. Hum Pathol. 1997;28(5):540-3. https://doi.org/10.1016/s00468177(97)90075-7.

12. Wu P, Zhang N, Wang $X$, Ning $X, L i T$, Bu D, et al. Family history of von Hippel-Lindau disease was uncommon in Chinese patients: suggesting the higher frequency of de novo mutations in $\mathrm{VHL}$ gene in these patients. J Hum Genet. 2012;57(4):238-43. https://doi.org/10.1038/jhg.2012.10.

13. Ebenazer A, Rajaratnam S, Pai R. Detection of large deletions in the VHL gene using a real-time PCR with SYBR green. Familial Cancer. 2013;12(3): 519-24.

14. Lundin P, Pedersen F. Volume of pituitary macroadenomas: assessment by MRI. J Comput Assist Tomogr. 1992;16(4):519-28. https://doi.org/10.1097/ 00004728-199207000-00004.

15. Butman JA, Linehan WM, Lonser RR. Neurologic manifestations of von Hippel-Lindau disease. JAMA. 2008;300(11):1334-42. https://doi.org/10.1001/ jama.300.11.1334.

16. Feletti A, Anglani M, Scarpa B, Schiavi F, Boaretto F, Zovato S, et al. Von Hippel-Lindau disease: an evaluation of natural history and functional. disability. Neuro-Oncology. 2016;18(7):1011-20. https://doi.org/10.1093/ neuonc/nov313.

17. Franke G, Bausch B, Hoffmann MM, Cybulla M, Wilhelm C, Kohlhase J, et al. Alu-Alu recombination underlies the vast majority of large VHL germline deletions: molecular characterization and genotype-phenotype correlations in VHL patients. Hum Mutat. 2009;30(5):776-86.

18. Yang C, Asthagiri AR, lyer RR, Lu J, Xu DS, Ksendzovsky A, et al. Missense mutations in the NF2 gene result in the quantitative loss of merlin protein and minimally affect protein intrinsic function. Proc Natl Acad Sci U S A. 2011;108(12):4980-5

19. Lu J, Yang C, Chen M, Ye DY, Lonser RR, Brady RO, et al. Histone deacetylase inhibitors prevent the degradation and restore the activity of glucocerebrosidase in Gaucher disease. Proc Natl Acad Sci U S A. 2011: 108(52):21200-5.

20. Maranchie JK, Afonso A, Albert PS, Kalyandrug S, Phillips JL, Zhou S, et al. Solid renal tumor severity in von Hippel Lindau disease is related to germline deletion length and location. Hum Mutat. 2004;23(1):40-6.

21. Aronoff L, Malkin D, van Engelen K, Gallinger B, Wasserman J, Kim RH, et al. Evidence for genetic anticipation in vonHippel-Lindau syndrome. J Med Genet. 2018;55(6):395-402.

22. Wang J, Peng $X$, Chen C, Ning X, Peng S, Li T, et al. Intra-familial phenotypic heterogeneity and telomere abnormality in von Hippel- Lindau disease: implications for personalized surveillance plan and pathogenesis of $\mathrm{VHL}-$ associated tumors. Front Genet. 2019;10:358. https://doi.org/10.3389/fgene. 2019.00358

23. Huntoon $\mathrm{K}$, Wu T, Elder JB, Butman JA, Chew EY, Linehan WM, et al. Biological and clinical impact of hemangioblastoma-associated peritumoral cysts in von Hippel-Lindau disease. J Neurosurg. 2016;124(4):971-6. https:// doi.org/10.3171/2015.4.JNS1533.

24. Berkman RA, Merrill MJ, Reinhold WC, Monacci WT, Saxena A, Clark WC et al. Expression of the vascular permeability factor/vascular endothelial growth factor gene in central nervous system neoplasms. J Clin Invest. 1993;91(1):153. 
25. Kano H, Shuto T, Iwai Y, Sheehan J, Yamamoto M, McBride HL, et al. Stereotactic radiosurgery for intracranial hemangioblastomas: a retrospective international outcome study. J Neurosurg. 2015;122(6):146978. https://doi.org/10.3171/2014.10.jns131602.

26. Ampie L, Choy W, Lamano JB, Kesavabhotla K, Kaur R, Parsa AT, et al. Safety and outcomes of preoperative embolization of intracranial

hemangioblastomas: a systematic review. Clin Neurol Neurosurg. 2016;150: 143-51. https://doi.org/10.1016/j.clineuro.2016.09.008.

27. Cornelius JF, Saint-Maurice JP, Bresson D, George B, Houdart E. Hemorrhage after particle embolization of hemangioblastomas: comparison of outcomes in spinal and cerebellar lesions. J Neurosurg. 2007;106(6):994-8. https://doi. org/10.3171/jns.2007.106.6.994.

28. Takeuchi S, Tanaka R, Fujii Y, Abe H, Ito Y. Surgical treatment of hemangioblastomas with presurgical endovascular embolization. Neurol Med Chir (Tokyo). 2001;41(5):246-51; discussion 51-2. https://doi.org/10. 2176/nmc.41.246.

\section{Publisher's Note}

Springer Nature remains neutral with regard to jurisdictional claims in published maps and institutional affiliations.

Ready to submit your research? Choose BMC and benefit from:

- fast, convenient online submission

- thorough peer review by experienced researchers in your field

- rapid publication on acceptance

- support for research data, including large and complex data types

- gold Open Access which fosters wider collaboration and increased citations

- maximum visibility for your research: over $100 \mathrm{M}$ website views per year

At BMC, research is always in progress.

Learn more biomedcentral.com/submissions 